\title{
Estudio del comportamiento en compras de clientes de Manabí en una empresa de retail un año después del terremoto
}

\section{Study of the behavior in purchases of Manabí clients in a retail company one year after the earthquake}

Hugo Renán Ruiz López

Universidad Internacional del Ecuador, Ecuador

Autor por correspondencia: huruizlo@uide.edu.ec

Fecha de recepción: 08 de Junio de 2017 - Fecha de aceptación: 10 de Agosto de 2017

\section{Resumen}

En el presente trabajo se estudia el comportamiento de compras en una tienda de retail de los clientes de la provincia de Manabí luego del desastre natural suscitado el 16 de abril del 2016, por ello analizamos las compras un año antes y un año después del terremoto, determinamos si existen variación en las compras de un periodo a otro, y adicionalmente segmentamos los clientes de la tienda en función de su variación en compras.

Palabras clave: terremoto, manabí; variación en compras; perfiles de clientes

\begin{abstract}
In the present work we study the behavior of purchases in a retail store of the customers of the province of Manabí after the natural disaster that arose on April 16, 2016, so we analyze the purchases a year before and a year after earthquake, we determine if there is a variation in purchases from one period to another, and additionally segment customers of the store based on their variation in purchases.
\end{abstract}

Key words: earthquake, manabí; variation in purchasing; customer profiles 


\section{Introducción}

El 16 de abril, un terremoto de 7,8 grados de magnitud sacudió la zona costera de nuestro país y devastó a varios poblados y ciudades de la costa, en especial de Manabí y el sur de Esmeraldas (Profitas, s.f.). Este desastre natural generó la muerte de 660 personas, 32 desaparecidos y 22.421 personas albergadas (Secretaria Nacional de Gestión de Riesgo, 2016). En Manabí hubo 646 personas fallecidas, siendo los cantones con mayor afectación Manta (210 fallecidos), Pedernales (173 fallecidos) y Portoviejo con 133 fallecidos. (Breilh \& Zamora, 2017).

El terremoto sucedió un sábado en horas de la tarde, de haber ocurrido en días laborables se estimada un mayor número de afectados.

La falta inicial de información ocasionó miedo e incertidumbre en los ecuatorianos, luego fueron apareciendo las primeras imágenes y testimonios de los afectados. Se trataba de uno de los peores desastres que había ocurrido en el país en los últimos años.

La población afectada, en su mayoría, ha sido desplazada de sus lugares de origen, lo que ha generado migraciones internas, dirigidas especialmente a las grandes ciudades y de esta manera han venido a alimentar los cinturones de pobreza (Breilh \& Zamora, 2017).

Se calcula que se requerirán \$ 3.344 millones para solventar los gastos de reconstrucción. De este monto, el 67\% (\$ 2.253 millones) será asumido por el sector público y el 33\% (1.091 millones) por el sector privado (El Telégrafo, 2016).

Con una afectación al 49\% del sector productivo y con pérdidas por al menos $\$ 800$ millones, según datos del Ministerio de Industrias y Productividad (El Universo, 2016), aun los efectos siguen latentes en las economías de Manabí y Esmeraldas.

Más de un año de la tragedia se han reconstruido escuelas, carreteras, centros de salud, infraestructura eléctrica y de suministro de agua potable.

Se han utilizado alrededor de \$ 2.410 millones de dólares (Metro Ecuador, 2017) para la construcción de viviendas, construcción y adecuación de escuelas, construcción y restauración de hospitales y para la reparación en vialidad.

\section{Situación Económica de Manabí}

El terremoto ocurrido el 16 de abril, sin dudas creo un cambio en la vida de todos los ecuatorianos, en especial los que habitan en las provincias de Manabí y Esmeraldas. La destrucción ocasionada por el desastre agravó aún más la situación productiva, laboral y económica que ya estaba viviendo el país antes del terremoto.

De acuerdo al último censo poblacional y vivienda del INEC, Manabí cuenta con 1,369.780 habitantes, la tercera provincia más poblada del país. En el 2015 según el censo de empresas y establecimientos realizados por el mismo INEC, Manabí cuenta con 67.891 empresas (INEC, Directorio de Empresas - DIEE 2015_v2), de las cuales 93.3\% son microempresas. 
Tabla 1: Segmentos de Empresas

EMPRESAS EN MANABÍ

\begin{tabular}{|l|r|r|}
\hline Tamaño Empresa & \# Establecimientos & \% Part. \\
\hline Microempresa & 63.720 & $93,9 \%$ \\
\hline Pequeña empresa & 3.378 & $5,0 \%$ \\
\hline Mediana empresa "A" & 372 & $0,5 \%$ \\
\hline Mediana empresa "B" & 232 & $0,3 \%$ \\
\hline Grande empresa & 189 & $0,3 \%$ \\
\hline Total & $\mathbf{6 7 . 8 9 1}$ & $\mathbf{1 0 0 , 0 \%}$ \\
\hline
\end{tabular}

Fuente: Directorio de Empresas - DIEE 2015_v2 INEC

A continuación presentamos la clasificación de las empresas de acuerdo a su volumen de ventas y personal que labora.

Tabla 2: Clasificación de las empresas

\begin{tabular}{|l|l|l|}
\hline Clasificación de las empresas & Volúmenes de ventas anuales & Personal ocupado \\
\hline Micro empresa & Menor a 100.000 & 1 A 9 \\
\hline Pequeña empresa & De 100.001 a 1'000.000 & 10 A 49 \\
\hline Mediana empresa "A" & De 1'000.001 a 2'000.000 & 50 A 99 \\
\hline Mediana empresa "B" & De 2'000.001 a 5'000.000 & 100 A 199 \\
\hline Grande empresa & De 5'000.001 en adelante & 200 en adelante \\
\hline
\end{tabular}

Fuente: Directorio de Empresas - DIEE 2015_v2 INEC

Nota: Estratos de Ventas, según la Comunidad Andina de Naciones. Decisión 702, Articulo 3.

Estas empresas en el 2015 vendieron \$ 4.718 millones de dólares, que equivale al $2.9 \%$ de las ventas a nivel nacional (INEC, Directorio de Empresas - DIEE 2015_v2). Los sectores que más aportaron a las ventas fueron el Comercio y la Industria Manufacturera con el 36\% y 35\% del total de ventas.

Tabla 3: Ventas 2015 por sector económico

\begin{tabular}{|l|rr|r|}
\hline \multicolumn{1}{|c|}{ Sectores Económicos 2015} & \multicolumn{2}{|c|}{ ventas totales } & \multicolumn{2}{c|}{$\%$ Part. } \\
\hline Comercio & $\$$ & 1.694 & $36 \%$ \\
Industrias Manufactureras & $\$$ & 1.663 & $35 \%$ \\
Servicios & $\$$ & 699 & $15 \%$ \\
Agricultura, ganadería, silvicultura y pesca & $\$$ & 455 & $10 \%$ \\
Construcción & $\$$ & 194 & $4 \%$ \\
Explotación de Minas y Canteras & $\$$ & 13 & $0 \%$ \\
\hline Total & $\$$ & $\mathbf{4 . 7 1 8}$ & $\mathbf{1 0 0 \%}$ \\
\hline
\end{tabular}

Fuente: Directorio de Empresas - DIEE 2015_v2 INEC

Ventas en millones 
El sector de comercio es donde radican mayoritariamente los negocios de las empresas, excepto las Grande Empresas donde la mayor proporción es en el sector de servicios.

Tabla 4: Sector económico y clasificación de empresas

\begin{tabular}{|c|c|c|c|c|c|}
\hline Sectores Económicos 2015 & Microempresa & $\begin{array}{l}\text { Pequeña } \\
\text { empresa }\end{array}$ & $\begin{array}{c}\text { Mediana } \\
\text { empresa "A" }\end{array}$ & $\begin{array}{c}\text { Mediana } \\
\text { empresa "B" }\end{array}$ & $\begin{array}{l}\text { Grande } \\
\text { empresa }\end{array}$ \\
\hline Agricultura, ganadería, silvicultura y pesca & $17 \%$ & $10 \%$ & $8 \%$ & $11 \%$ & $9 \%$ \\
\hline Explotación de Minas y Canteras & $0 \%$ & $0 \%$ & $0 \%$ & $0 \%$ & $1 \%$ \\
\hline Industrias Manufactureras & $8 \%$ & $6 \%$ & $5 \%$ & $6 \%$ & $17 \%$ \\
\hline Comercio & $37 \%$ & $43 \%$ & $51 \%$ & $49 \%$ & $28 \%$ \\
\hline Construcción & $3 \%$ & $5 \%$ & $4 \%$ & $3 \%$ & $4 \%$ \\
\hline Servicios & $34 \%$ & $36 \%$ & $31 \%$ & $31 \%$ & $41 \%$ \\
\hline Total & $100 \%$ & $100 \%$ & $100 \%$ & $100 \%$ & $100 \%$ \\
\hline
\end{tabular}

Fuente: Directorio de Empresas - DIEE 2015_v2 INEC

Los cantones de Portoviejo y Manta concentran el mayor número de empresas de la provincia con el 48\%, que equivale a 32.653 empresas (INEC, Directorio de Empresas - DIEE 2015_v2). Adicionalmente el 58\% de las grandes empresas se encuentran en esos dos cantones (INEC, Directorio de Empresas - DIEE 2015_v2), esto equivale a 111 empresas grandes de 189 existentes en la provincia.

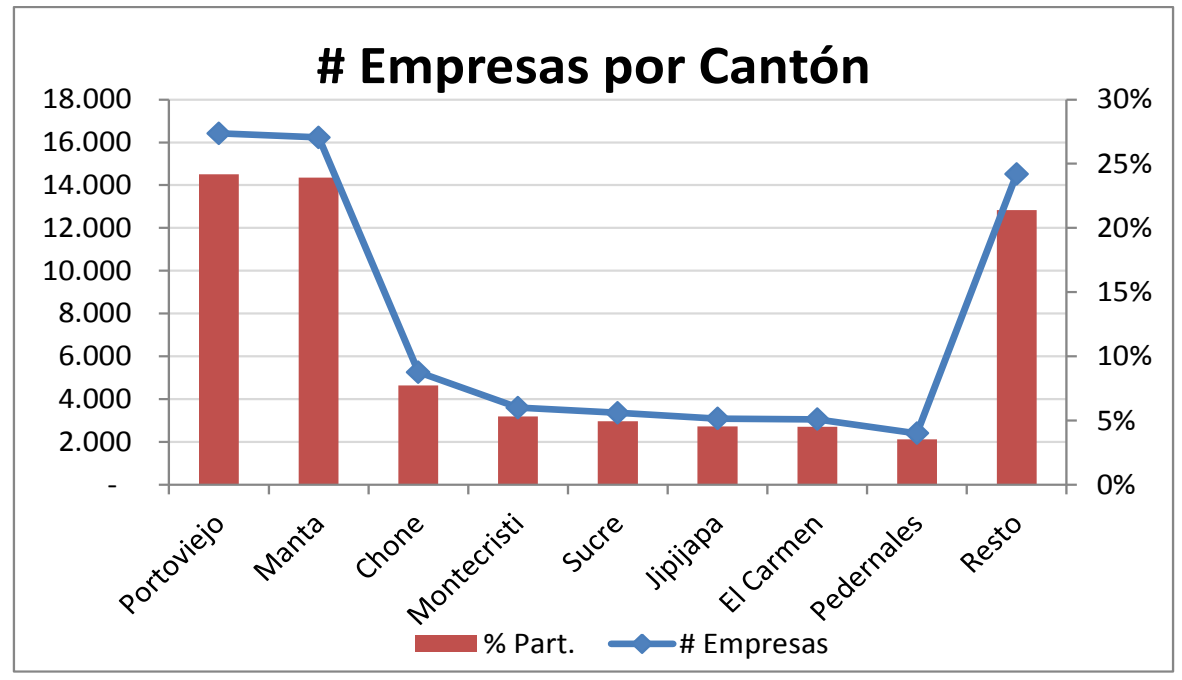

Gráfico 1: Número de empresas por cantones

El personal empleado y afiliado al IESS en el 2015 fue de 162.665 personas (INEC, Directorio de Empresas - DIEE 2015_v2), de los cuales el 58\% fueron hombres y el $42 \%$ mujeres. En el cantón de Junín existe la mayor diferencia de hombres empleados con relación a las mujeres, de las 1.203 personas empleadas, el 74\% son hombres y el $26 \%$ mujeres. Mientras que en el cantón Santa Ana la proporción de hombres y mujeres empleados es prácticamente similar, 2.980 empleados, $51 \%$ son hombres y $49 \%$ mujeres. 
El impacto económico del terremoto es muy alto, pues aproximadamente el $11 \%$ de las empresas y negocios está afectado (Opción Quincenario). Luego del terremoto se despidieron a 30.000 trabajadores en el sector comercial, de servicios y manufacturero principalmente (Opción Quincenario).

La pérdida de plazas de trabajo se ha visto reflejada en el aumento de solicitudes del seguro de desempleo IESS. Hasta antes del terremoto apenas 37 personas habían pedido el beneficio. Desde el 16 de abril esa cifra se elevó a 1.552 en Manabí (El Universo, 2016).

Del sector productivo, el comercio fue el más afectado por el desastre, con pérdidas por \$ 428 millones, refiere el ministro de industrias de ese momento Santiago León (El Universo, 2016). En agricultura y pesca la afectación fue de $\$ 220$ millones, mientras que en manufactura la afectación fue de \$ 164 millones.

Otra de las afectaciones son también las exportaciones, según la Federación Ecuatoriana de Exportadores (Fedexpo), 150 millones se dejarán de exportar por el terremoto, luego de analizar la producción, infraestructura y logística de las firmas exportadoras que se asienta en Manabí y Esmeraldas (Revista Líderes, 2016).

Los bienes patrimoniales también sufrieron afectaciones por el terremoto, de 323 bienes inventariados, el 63\% tuvieron alguna afectación (Diario La Hora, 2016).

\section{Variación de las compras de clientes de Manabí en una empresa de Retail después del terremoto}

Se trabajó con una muestra aleatoria de 943 clientes con domicilio en la provincia de Manabí, que realizaron compras en el periodo comprendido entre Abril 2015 y Marzo 2016 “Antes del Terremoto" en una empresa de Retail en la ciudad de Guayaquil. Luego consideramos las compras de estos clientes en el periodo de Abril 2016 hasta Marzo 2017 "Después del Terremoto", para realizar las respectivas comparaciones.

Adicionalmente seleccionamos aleatoriamente 2.977 clientes de la misma empresa de Retail pero domiciliados fuera de la provincia de Manabí. A continuación presentamos los principales resultados.

Variación en compras: Los clientes de Manabí realizaron compras por \$ 326 mil dólares en el periodo posterior al terremoto, $29 \%$ menos que las compras registradas en el periodo anterior al terremoto que fueron de \$ 451 mil dólares. El 33\% de los clientes que compraron en el periodo antes del terremoto no volvieron a comprar después del mismo.

A nivel nacional las ventas disminuyeron un $10 \%$ después del terremoto en comparación con las registradas antes del desastre, mientras que el 17\% de los clientes dejaron de comprar. 


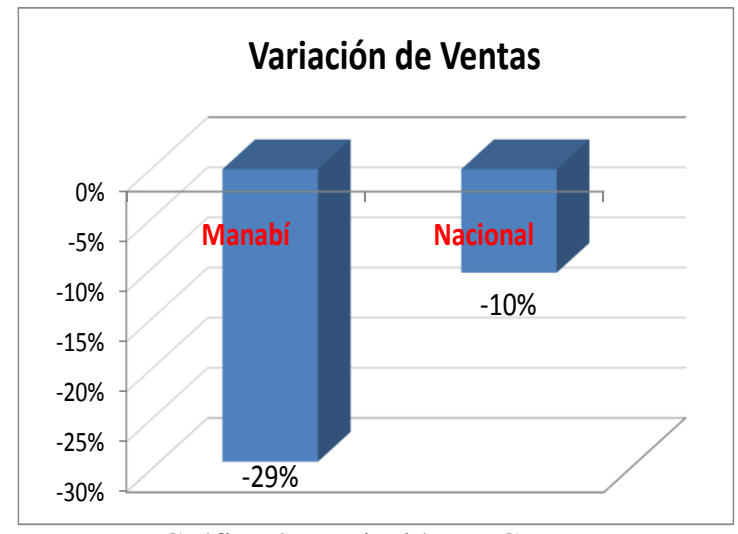

Gráfico 2: Variación en Compras

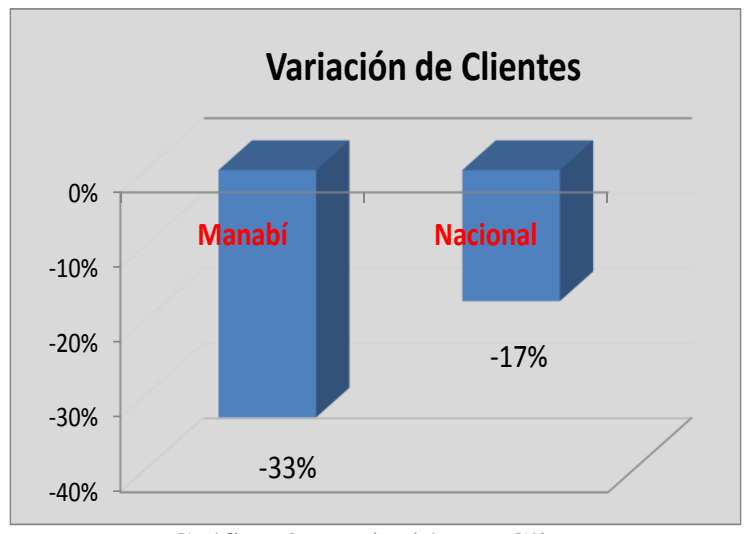

Gráfico 3: Variación en Clientes

Edad del Cliente: La edad promedio de los clientes de Manabí es de 40,7 años, siendo su edad mínima de 20 años y máxima de 78 años. Los clientes mayores a 40 años registraron una disminución en ventas superior al promedio que fue del $29 \%$.

Tabla 5: Compras por Periodo y Rango de Edad

\begin{tabular}{|l|lr|lr|r|}
\hline \multirow{2}{*}{ Rango Edad } & \multicolumn{4}{|c|}{ Compras } & \multirow{2}{*}{$\%$ var. } \\
\cline { 2 - 4 } & Antes Terrem. & Después Terrm. & \\
\hline $20-29$ & $\$$ & 61.543 & $\$$ & 46.247 & $-25 \%$ \\
$30-39$ & $\$$ & 147.876 & $\$$ & 112.008 & $-24 \%$ \\
$40-49$ & $\$$ & 129.171 & $\$$ & 84.121 & $-35 \%$ \\
$>49$ & $\$$ & 112.746 & $\$$ & 77.284 & $-31 \%$ \\
\hline Total & $\mathbf{\$}$ & $\mathbf{4 5 1 . 3 3 6}$ & $\mathbf{\$}$ & $\mathbf{3 1 9 . 6 5 9}$ & $\mathbf{- 2 9 \%}$ \\
\hline
\end{tabular}

Sexo del Cliente: El 74\% de los clientes de Manabí son del sexo femenino, mientras que el $26 \%$ de sexo masculino. Los clientes de sexo masculino disminuyeron sus compras en un $41 \%$ después del terremoto, 16 puntos más que los clientes de sexo femenino que obtuvieron una disminución del $24 \%$ en sus compras con relación al periodo antes del desastre natural.

Tabla 6: Compras por Periodo y Sexo del Cliente

\begin{tabular}{|c|c|c|c|c|c|}
\hline \multirow{2}{*}{ Sexo } & \multicolumn{4}{|c|}{ Compras } & \multirow{2}{*}{$\%$ var. } \\
\hline & An & s Terrem. & $\mathrm{De}$ & és Terrm. & \\
\hline Femenino & $\$$ & 342.442 & $\$$ & 255.412 & $-25 \%$ \\
\hline Masculino & $\$$ & 108.894 & $\$$ & 64.247 & $-41 \%$ \\
\hline Total & $\$$ & 451.336 & $\$$ & 319.659 & $-29 \%$ \\
\hline
\end{tabular}

Ciudad Domicilio: Las ciudades de Portoviejo y Manta registran el mayor número de clientes con el $33 \%$ y $29 \%$ respectivamente, le siguen con menor proporción Jipijapa, Chone y Bahía de Caráquez con 7\%, 6\% y 5\%.

Portoviejo que fue una de las ciudades más golpeadas por el terremoto, tiene la mayor disminución en compras con 38\%, 8 puntos superior al promedio de la provincia. Mientras que Manta registro una disminución del 21\%,8 puntos inferior al promedio.

Tabla 7: Compras por Periodo y Ciudad Domicilio 


\begin{tabular}{|l|lr|lr|r|}
\hline \multirow{2}{*}{$\begin{array}{c}\text { Ciudad } \\
\text { Domicilio }\end{array}$} & \multicolumn{4}{|c|}{ Compras } & \multirow{2}{*}{$\%$ var. } \\
\cline { 2 - 5 } & Antes Terrem. & Después Terrm. & \\
\hline MANTA & $\$$ & 130.009 & $\mathbf{S}$ & 102.089 & $-21 \%$ \\
PORTOVIEJO & $\$$ & 151.290 & $\mathbf{S}$ & 94.001 & $-38 \%$ \\
RESTO & $\$$ & 170.037 & $\mathbf{\$}$ & 123.569 & $-27 \%$ \\
\hline Total & $\mathbf{\$}$ & $\mathbf{4 5 1 . 3 3 6}$ & $\mathbf{\$}$ & $\mathbf{3 1 9 . 6 5 9}$ & $\mathbf{- 2 9 \%}$ \\
\hline
\end{tabular}

\section{Perfil de los Clientes que más disminuyeron las compras después de terremoto}

Mediante la metodología de árboles de decisión se han obtenido los siguientes perfiles de clientes con base a su disminución de compras posterior al terremoto. A continuación presentamos los perfiles de los clientes y sus respectivas compras antes y después del terremoto:

Segmentamos los clientes de Manabí en 7 segmentos, los cuatro primeros segmentos contiene una disminución en compras superior al promedio de la provincia que fue del $29 \%$, de estos los tres primeros tienen la característica que el domicilio es diferente de Manta.

Los clientes domiciliados en Manta que más disminuyeron sus ventas fueron los del sexo masculino con el $36 \%$ de decrecimiento.

Aquellos clientes con domicilio en Manta, de sexo femenino y mayores a 36 años registraron el $13 \%$ de reducción en compras luego del terremoto, mientras que aquellos clientes no domiciliados en Manta que tuvieron una menor disminución en sus compras fueron del sexo femenino y menores a 38 años con el $16 \%$ de contracción en compras.

Tabla 8: Segmentos de Clientes de acuerdo a su variación en Compras

\begin{tabular}{|c|c|c|c|c|c|c|}
\hline \multirow{2}{*}{ Segmento } & \multirow{2}{*}{ Perfil del Cliente } & \multicolumn{4}{|c|}{ Compras } & \multirow{2}{*}{$\%$ var. } \\
\hline & & \multicolumn{4}{|c|}{ Antes Terrem. Después Terrm. } & \\
\hline 1 & Maculino, Domicilio diferente de Manta, edad <= 34 años & $\$$ & 18.861 & $\$$ & 8.584 & $-54 \%$ \\
\hline 2 & Maculino, Domicilio diferente de Manta, edad > 34 años & $\$$ & 52.981 & $\$$ & 31.847 & $-40 \%$ \\
\hline 3 & Femenino, Domicilio diferente de Manta, edad >38 años & $\$$ & 147.043 & $\$$ & 91.527 & $-38 \%$ \\
\hline 4 & Masculino, Domicilio Manta & $\$$ & 37.051 & $\$$ & 23.816 & $-36 \%$ \\
\hline 5 & Femenino, Domicilio Manta, edad $<=36$ años & $\$$ & 40.741 & $\$$ & 32.872 & $-19 \%$ \\
\hline 6 & Femenino, Domicilio diferente de Manta, edad <= 38 años & $\$$ & 102.442 & $\$$ & 85.612 & $-16 \%$ \\
\hline 7 & Femenino, Domicilio Manta, edad >36 años & $\$$ & 52.216 & $\$$ & 45.401 & $-13 \%$ \\
\hline \multicolumn{2}{|r|}{ Total } & $\$$ & 451.336 & $\$$ & 319.659 & $-29 \%$ \\
\hline
\end{tabular}

El siguiente gráfico nos muestra la participación de clientes por segmento, como se puede apreciar el segmento 1 es el más pequeño en tamaño de clientes con el $6 \%$, mientras que el segmento 3 es el de mayor tamaño con el $28 \%$ de participación. 


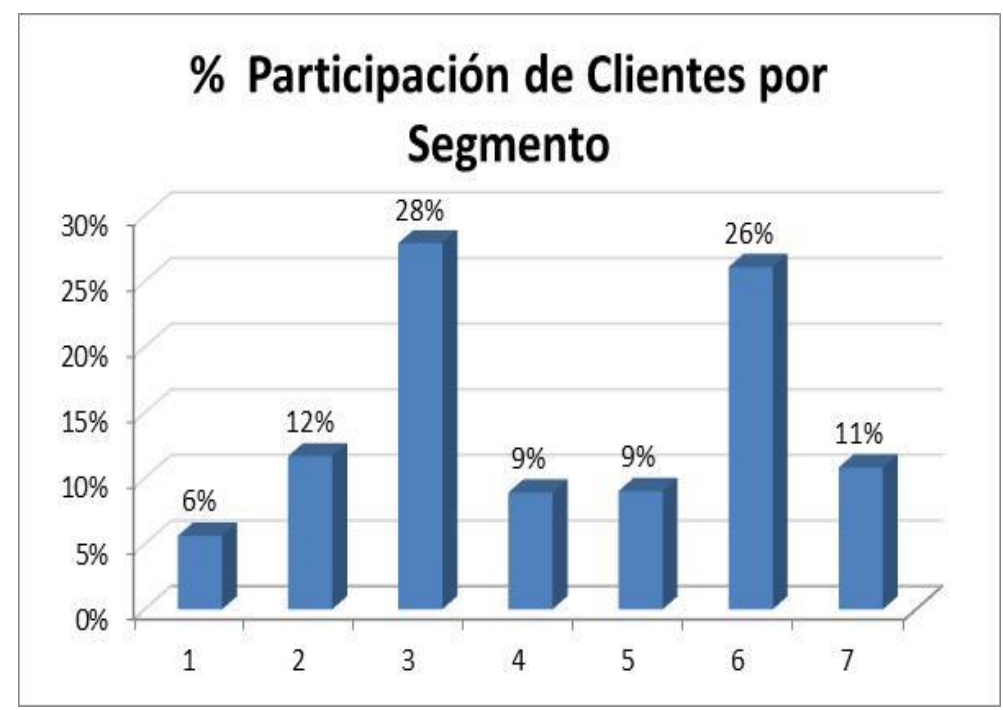

Gráfico 4: Porcentaje de Participación de Clientes por Segmentos

\section{Conclusiones}

Los clientes de Manabí disminuyeron sus compras un 29\% posterior al terremoto, mientras que la disminución del resto del país fue del $10 \%$.

Un 33\% de los clientes de Manabí que compraron antes del terremoto dejaron de comprar en el periodo después del sismo, a nivel nacional los clientes que no volvieron a comprar luego del sismo fue del $17 \%$.

En Manabí los hombres disminuyeron sus compras en un $41 \%$, mientras que la disminución en las mujeres fue del 25\%.

La ciudad de Portoviejo una de las afectadas por terremoto, fue la que más disminuyó sus compras luego del desastre natural con el $38 \%$ menos de gasto.

Los hombres, con ciudad domicilio diferente de Manta y menores a 34 años, han obtenido la mayor disminución en compras con el 54\%, estos clientes representan el $6 \%$ de la muestra en estudio.

Las mujeres, con domicilio en Manta y mayores a 36 años, registraron la menor disminución en compras con el 13\%, este segmento representa el $11 \%$ de la muestra.

\section{Bibliografía}

Breilh, J., \& Zamora, G. (2017). El Terremoto de abril, y la determinación social de la vulnerabilidad. uasb.

Diario La Hora. (15 de Mayo de 2016). Diario La Hora. Obtenido de https://lahora.com.ec/noticia/1101944588/bienes-patrimoniales--afectados-por-sismo-enmanabc3ad- 
El Telégrafo. (2 de Junio de 2016). El Telégrafo.com.ec. Obtenido de http://www.eltelegrafo.com.ec/noticias/economia/8/usd-3-344-millones-es-el-costo-de-lareconstruccion-de-las-zonas-afectadas

El Universo. (26 de Julio de 2016). El comercio fue el más afectado tras el sismo de 7,8 en Ecuador. http://www.eluniverso.com/noticias/2016/07/29/nota/5714052/comercio-fue-masafectado-tras-sismo-78

INEC. (s.f.). Directorio de Empresas - DIEE 2015_v2. Obtenido de http://www.ecuadorencifras.gob.ec/directoriodeempresas/

Metro Ecuador. (15 de Abril de 2017). https://www.metroecuador.com.ec. Obtenido de https://www.metroecuador.com.ec/ec/noticias/2017/04/15/ano-terremoto-ecuador.html

MIDUVI. (2016). Ministerio de Desarrollo Urbano y Vivienda del Ecuador.

Opción Quincenario. (s.f.). periodico opción. Obtenido de periodico opción: http://www.periodicopcion.tk/index.php/component/content/article?id=353:manabiantes-y-despues-del-terremoto-de-16-a

Profitas. (s.f.). http://www.profitas.com/. Obtenido de http://www.profitas.com/

Revista Líderes. (20 de Mayo de 2016). Revista Líderes. Obtenido de http://www.revistalideres.ec/lideres/ecuador-impacto-terremoto-magapexportaciones.html

Secretaria Nacional de Gestión de Riesgo. (2016). Iinforme del 30 abril de 2016. Secretaria Nacional de Gestión de Riesgo, 20. 